\title{
Redescription of little-known gnaphosid spider Talanites strandi Spassky, 1940 (Aranei: Gnaphosidae) from Crimea
}

\section{Переописание малоизвестного вида Talanites strandi Spassky, 1940 (Aranei: Gnaphosidae) из Крыма}

\author{
Mykola M. Kovblyuk, Zoya A. Kastrygina \\ Н.М. Ковбцюк, 3.А. Кастрыгина
}

Zoology Department, V.I. Vernadsky Taurida National University, 4 Yaltinskaya str., Simferopol 95007, Ukraine. E-mail: kovblyuk@mail.ru; zoiac_21@mail.ru

Кафедра зоологии Таврического национального университета им. В.И.Вернадского, ул. Ялтинская 4, Симферополь 95007, Украина.

KEY WORDS: spiders, Talanites strandi, redescription, Crimea.

КЛЮЧЕВЫЕ СЛОВА: пауки, Talanites strandi, переописание, Крым.

ABSTRACT. Redescription of the little-known Gnaphosid, Talanites strandi Spassky, 1940, is provided, based on specimens from Crimea. This species is new to the fauna of Crimea and the record from the peninsula is the westernmost locality of $T$. strandi distribution. Illustrations, diagnosis, distribution and seasonal activity are presented.

РЕЗЮМЕ. По экземплярам из Крыма переописан малоизвестный вид Talanites strandi Spassky, 1940. T. strandi отмечается в Крыму впервые. Крым - самая западная часть ареала T. strandi. Приведены рисунки таксономических признаков, диагно3, сведения о распространении и сезонной динамике активности половозрелых особей.

\section{Introduction}

This paper continues our studies of Crimean gnaphosid spiders. So far 67 gnaphosid species belonging to 20 genera have been reported from the Peninsula [Kovblyuk, 2004, 2006; Kovblyuk \& Tuneva, 2009; Kovblyuk et al., 2009; Kovblyuk \& Nadolny, 2010]. During recent research several additional species to the Crimean fauna were found in the Karadag Nature Reserve. The goal of this paper is to rediscribe the little-known species, Talanites strandi Spassky, 1940, and to provide data on the distribution and seasonal dynamics of activity of its adults in Crimea.

\section{Material and Methods}

Specimens for this study were recently collected in Crimea. All specimens are deposited in the collection of Zoology Department, V.I. Vernadsky Taurida Na- tional University, Simferopol, Ukraine, curator M.M. Kovblyuk (TNU). Following abbreviations are used in the text: a - apical; $\mathrm{d}$ - dorsal; $\mathrm{pl}$ - prolateral; $\mathrm{rl}$ retrolateral; $\mathrm{v}$ - ventral.

Illustrations were made using both reflected and transmitted light microscopes. Illustrations of epigyne and bulbus were made after maceration in $20 \% \mathrm{KOH}$ solution. Coloration was described from specimens preserved in $75 \%$ ethanol with added glycerin ( $9: 1$ by volume). Leg and palp segments were measured after their separation from the cephalothorax. All measurements are in mm. All scale bars are equal $0.1 \mathrm{~mm}$ if not otherwise indicated.

\section{Talanites Simon, 1893}

Type species: T. fervidus Simon, 1893 from Egypt and Israel.

The genus contains 15 species, mostly from the Holarctic [Platnick, 2012]. Two species, T. atscharicus Mcheidze, 1946 from Caucasus and T. cavernicola Thorell, 1897 from Myanmar, are known by females only, and probably are misplaced.

Talanites can be distinguished from other gnaphosid genera by the following combination of characters: presence of two dorsal spines on tibia IV; posteriormedian eyes nearly circular (usually triangular in most gnaphosid genera); legs with scopulae; male palp with bent tibia, thick embolus and greatly elongated median apophysis; males lack a dorsal scutum [Platnick \& Ovtsharenko, 1991; Levy, 1999].

Talanites strandi Spassky, 1940

Figs 1-12.

T. s. Spassky, 1940: 353, pl. 7, f. $1\left(\sigma^{7}\right)$.

T. s.: Platnick \& Ovtsharenko, 1991: 119, f. 13-14 ( $\left.\sigma^{7}\right)$. 

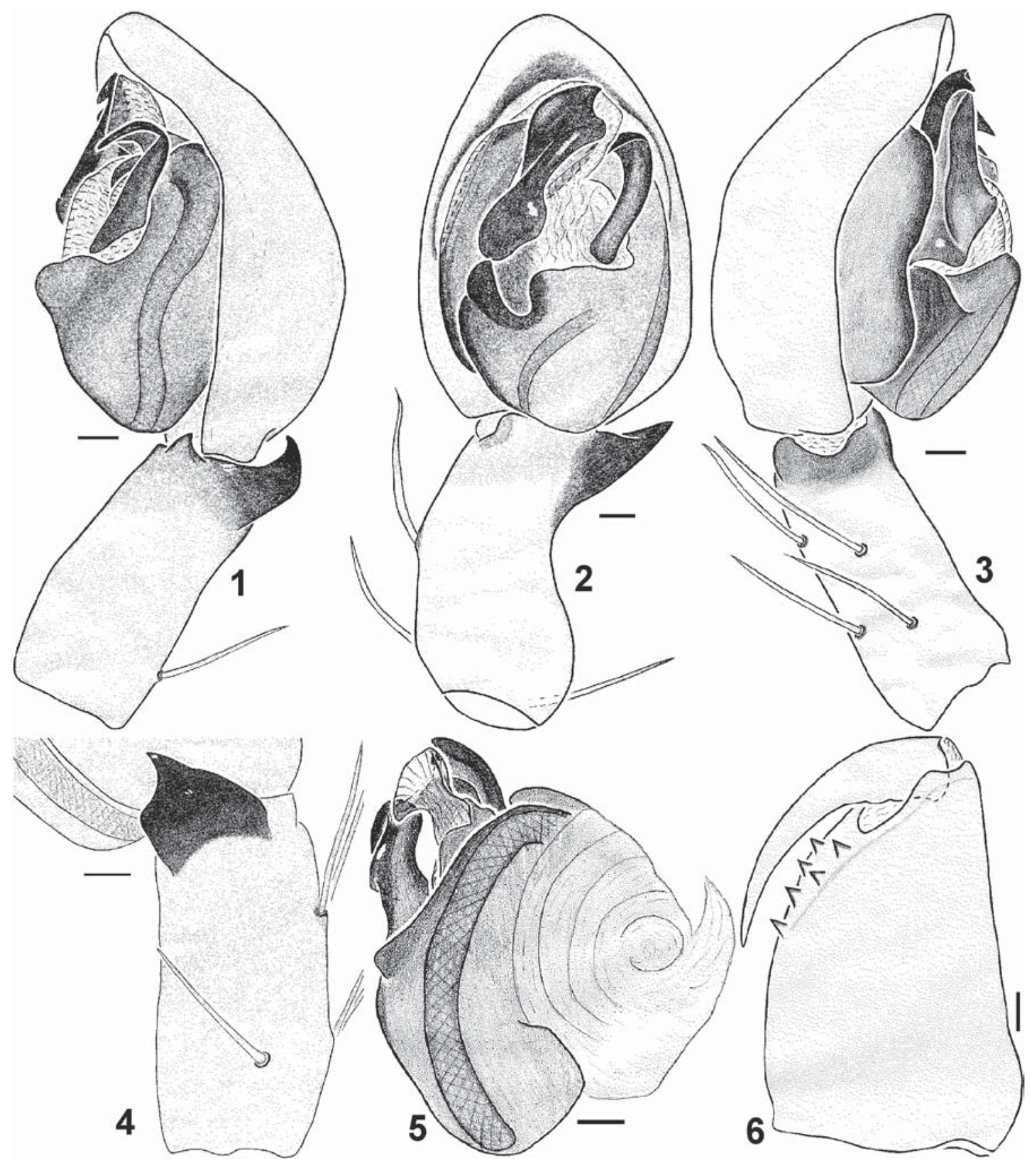

Figs 1-6. Male of Talanites strandi: 1 - palp, retrolateral view; 2 - palp, ventral view; 3 - palp, prolateral view; 4 - palpal tibia, retrolateral view; 5 - bulbus, dorso-retrolateral view; 6 - left chelicera, posterior view. Scale bars: $0.1 \mathrm{~mm}$.

Рис. 1-6. Самец Talanites strandi: 1 - пальпа, ретролатерально; 2 - пальпа, вентрально; 3 - пальпа, пролатерально; 4 голень пальпы, ретролатерально; 5 - бульбус, дорсо-ретролатерально; 6 - левая хелицера, сзади. Масштаб: 0,1 мм.

T. s.: Ponomarev \& Tsvetkov, 2004: 94, f. 9-10 (+).

T. s.: Wunderlich, 2011: 37, f. 30 (drawings taken from Platnick \& Ovtsharenko [1991]; $\sigma^{7}$ only; epigyne in f. 31 is misidentified refers to T. moodyae Platnick \& Ovtsharenko, 1991).

MATERIAL. UKRAINE. Crimea: Feodosiya Distr.: $8 \sigma^{\top} \sigma^{\top}$ (TNU-2910/6, 2911/6, 2912/2, 2913/6), Karadag Nature Reserve, N44 $4^{\circ} 4^{\prime} 44,9^{\prime}$ ' E $35^{\circ} 12^{\prime} 37,5^{\prime \prime}, 5 \mathrm{~m}$, sea shore, Pistacia mutica, Scumpia, pitfalls, 6.06.-4.08.2008, A.A. Nadolny. Sudak Distr.: 1 $\mathrm{O}^{\top}, 3$ 우 (TNU-2832/14, 2848/5), $10 \mathrm{~km}$ west from Sudak,
Mezhdurechie Vill., grassland, steppe, pitfalls, 2-17.07.2010, M.K. Yusufova.

DIAGNOSIS. Shape of embolus and tibial apophysis in male palp, and shape of the epigynal fovea are diagnostic characters that clearly separate $T$. strandi from all other congeners.

DESCRIPTION. Males $(\mathrm{n}=2)$ and females $(\mathrm{n}=2)$. Measurements (\#/\$): total length 7.3-9.0 / 7.9-8.1; 
carapace 3.4-4.3 / 3.4-3.6 long, 2.6-3.2 / 2.6-2.7 wide; abdomen 4.0-4.6 / 4.4-4.7 long, 2.05-2.9 / 2.45-2.7 wide. Diameters of eyes and interdistances: AM $0.12 /$ 0.09, AL 0.14 / 0.12-0.15, PM 0.10 / 0.10, PL 0.12 / 0.12-0.14, AM-AM 0.06-0.08/ 0.06-0.08, AM-AL 0.06-0.09 / 0.06, PM-PM 0.14 / 0.16, PM-PL 0.14 / 0.14-0.15, AM-PM 0.08-0.09 / 0.08-0.10, AL-PL 0.06-0.08/ 0.06-0.08. Distances between anterior eyes and margin of clypeus: ÀM-clypeus $0.14-0.18$ / 0.09 0.10 , AL-clypeus $0.10-0.15 / 0.06-0.10$. Length of palp segments (male/female): femur 1.1-1.2 / 1.1-1.3, patella $0.5-0.8 / 0.7$, tibia $0.7-0.8 / 0.7$, tarsus $1.1-1.2 /$ 1.0-1.1. Length of leg segments (male/female):

\begin{tabular}{|c|l|l|l|l|l|l|}
\hline Leg & \multicolumn{1}{|c|}{ Femur } & \multicolumn{1}{|c|}{ Patella } & \multicolumn{1}{|c|}{ Tibia } & \multicolumn{1}{|c|}{ Metatarsus } & \multicolumn{1}{|c|}{ Tarsus } & \multicolumn{1}{|c|}{ Total } \\
\hline \multirow{2}{*}{ I } & $2.5-3.0 /$ & $1.6-2.1 /$ & $2.5-3.0 /$ & $2.0-2.4 /$ & $1.4-1.8 /$ & $10.1-12.4 /$ \\
& $2.0-2.6$ & $1.1-1.7$ & $1.6-2.4$ & $1.3-1.8$ & $1.0-1.2$ & $7.0-10.0$ \\
\hline \multirow{2}{*}{ II } & $2.4-3.0 /$ & $1.5-2.0 /$ & $2.2-2.6 /$ & $1.8-2.3 /$ & $1.3-1.5 /$ & $92-11.5 /$ \\
& $2.2-2.5$ & $1.5-1.7$ & $2.0-2.1$ & $1.5-1.6$ & 1.1 & $8.2-8.9$ \\
\hline \multirow{2}{*}{ III } & $2.2-2.8 /$ & $1.3-1.8 /$ & $1.9-2.2 /$ & $2.0-2.3 /$ & $1.1-1.4 /$ & $8.5-105 /$ \\
& $2.1-2.3$ & 1.3 & $1.7-1.8$ & $1.7-1.9$ & $1.0-1.1$ & $79-8.4$ \\
\hline \multirow{2}{*}{ IV } & $2.8-3.3 /$ & $1.6-2.0 /$ & $2.6-3.0 /$ & $3.1-3.9 /$ & $1.4-1.7 /$ & $11.4-13.8 /$ \\
& $2.6-2.9$ & $1.5-1.7$ & $2.4-2.5$ & $2.8-3.1$ & $1.3-1.4$ & $10.6-11.5$ \\
\hline
\end{tabular}

Cheliceral teeth $\left(\sigma^{\top} / 9\right)$ : anterior -3 or $4 / 3$; posterior $-2 / 2$.

Male leg spination:

\begin{tabular}{|c|c|c|c|c|}
\hline & Femur & Patella & Tibia & Metatarsus \\
\hline Leg I & $\begin{array}{l}\text { d 1-1-1, } \\
\text { pl 1-1-1, } \\
\text { rl 1-1 }\end{array}$ & 0 & $\begin{array}{l}\text { pl 1-1-1, } \\
\text { rl 1-1, } \\
\text { v 1-1-2-2a }\end{array}$ & $\begin{array}{l}\text { pl 1, } \\
\text { rl 1, } \\
\text { v 2-2 }\end{array}$ \\
\hline Leg II & $\begin{array}{l}\text { d } 1-1-1 \text { or } 1-1 \text { ( } 1 \text { of } \\
2 \text { specimens), } \\
\text { pl } 1-1 \text {, } \\
\text { rl } 1-1 \text { or } 1-1-2 \text { (1 of } \\
2 \text { specimens) }\end{array}$ & 0 & $\begin{array}{l}\text { pl 1-1-1, } \\
\text { rl 1-1-1, } \\
\text { v 1-1-2-2a }\end{array}$ & $\begin{array}{l}\text { pl } 1 \text { or } 1-1 \text { (1 of } 2 \\
\text { specimens), } \\
\text { rl 1, } \\
\text { v } 2-2\end{array}$ \\
\hline Leg III & $\begin{array}{l}\text { d } 1-1-1, \\
\text { pl } 1-1 \text {, } \\
\text { rl } 1-1 \text { or } 1-1-1 \text { (1 of } \\
2 \text { specimens) }\end{array}$ & rl 1 & $\begin{array}{l}\text { d 1, } \\
\text { pl 1-1-1-1, } \\
\text { Il 1-1-1-1, } \\
\text { v 2-2-2a }\end{array}$ & $\begin{array}{l}\text { pl 3-2-2, } \\
\text { rl 1-1-1-2, } \\
\text { v 2-2-2a }\end{array}$ \\
\hline Leg IV & $\begin{array}{l}\text { d 1-1-1, } \\
\text { pl 1-1-1, } \\
\text { rl 1-1-1 }\end{array}$ & rl 1 & $\begin{array}{l}\text { d 1-1, } \\
\text { pl 1-1-1-1, } \\
\text { rl 1-1-1-1, } \\
\text { v } 2-1-1-2 \mathrm{a} \text { or } \\
\text { 2-2-2a (1 of } 2 \\
\text { specimens) }\end{array}$ & $\begin{array}{l}\text { pl } 2-2-2, \\
\text { rl } 1-1-1-2, \\
\text { v } 2-1-1-2 \mathrm{a} \text { or } 2-1-2-2 \mathrm{a} \\
\text { ( } 1 \text { of } 2 \text { specimens) }\end{array}$ \\
\hline
\end{tabular}

Female leg spination:

\begin{tabular}{|c|c|c|c|c|}
\hline & Femur & Patella & Tibia & Metatarsus \\
\hline Leg I & $\begin{array}{l}\text { d 1-1-1, } \\
\text { pl 1-1-1 }\end{array}$ & 0 & $\begin{array}{l}\text { v } 1-1-2-2 \mathrm{a} \text { or } \\
2-1-1-1-1 \mathrm{a}(1 \text { of } 2 \\
\text { specimens) }\end{array}$ & v 2-2 \\
\hline Leg II & $\begin{array}{l}\text { d } 1-1, \\
\text { pl } 1-1, \\
\text { rl } 0 \text { or } 1-1 \text { ( } 1 \text { of } \\
2 \text { specimens) }\end{array}$ & 0 & v $1-1-2-2 a$ & v $2-2$ \\
\hline Leg III & $\begin{array}{l}\text { d 1-1-1, } \\
\text { pl 1-1, } \\
\text { rl 1-1-1 }\end{array}$ & $\mathrm{rl}$ & $\begin{array}{l}\text { d } 1 \text { or } 1-1 \text { (1 of } 2 \\
\text { specimens), } \\
\text { pl 1-1-1, } \\
\text { rl 1-1-1, } \\
\text { v 2-2-2a }\end{array}$ & $\begin{array}{l}\text { pl 3-2-2, } \\
\text { rl 1-1-1-2, } \\
\text { v 2-2-2a }\end{array}$ \\
\hline Leg IV & $\begin{array}{l}\text { d 1-1-1, } \\
\text { pl 1-1, } \\
\text { rl 1-1 }\end{array}$ & rl 1 & $\begin{array}{l}\text { d 1-1, } \\
\text { pl 1-1-1-1, } \\
\text { rl 1-1-1-1, } \\
\text { v 2-2-2a }\end{array}$ & $\begin{array}{l}\text { pl } 2-2-2, \\
\text { rl } 1-1-1-2 \text { or } 2-1-1-2 \\
\text { ( } 1 \text { of } 2 \text { specimens }) \\
\text { v } 2-1-1-2 \mathrm{a}\end{array}$ \\
\hline
\end{tabular}

In both sexes tarsi I-III and metatarsi I-II with scopula. Tarsi IV in both sexes are remarkably thin and arched, in contrast to unmodified tarsi I-III.

Coloration yellow with characteristic dorsal pattern on abdomen (Fig. 7).

Male palp with peculiar robust embolus with folded and invaginated tip, median apophysis with second point and broad retrolateral tibial apophysis (Figs 15). Epigyne with peculiar wide depression and massive spermathecae; hood is lacking (Figs 9-11).

VARIATION. Second point in median apophysis varies in size from large to very small (cf. Figs 1-3, 5 and fig. 14 in Platnick \& Ovtsharenko [1991]).

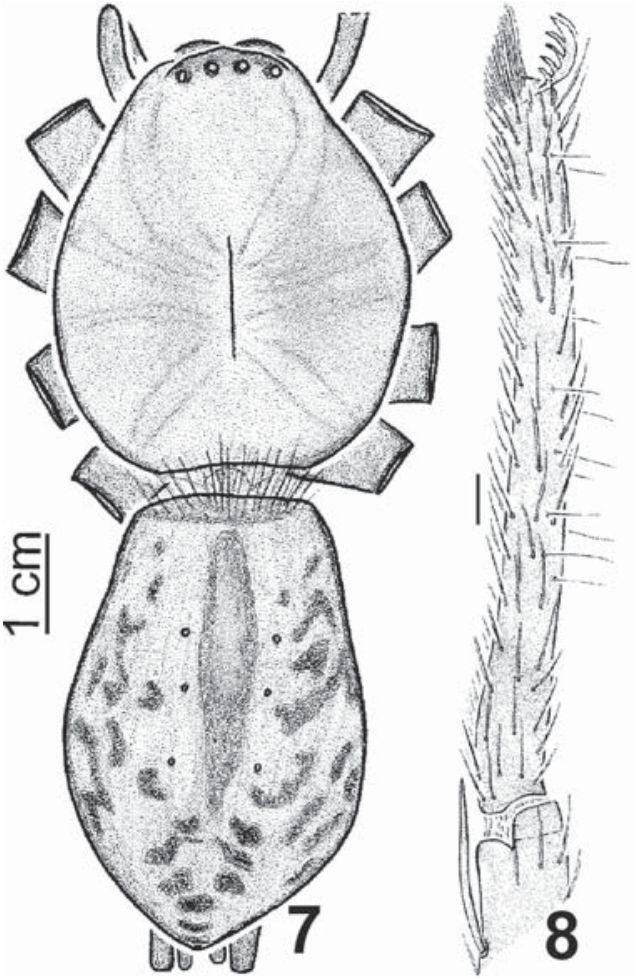

Figs 7-8. Male of Talanites strandi: 7 - general appearance, dorsal; 8 - tarsus IV, retrolateral view. Scale bar: $0.1 \mathrm{~mm}$.

Рис. 7-8. Самец Talanites strandi: 7 - габитус, дорсально; 8 - лапка IV, ретролательно. Масштаб: 0,1 мм.

TYPE LOCALITY. Amvrossievka Vill., Donetsk Area, Ukraine [Spassky, 1940: 355].

DISTRIBUTION. Ukraine (Crimea, Donetsk Area), Russia (Rostov and Volgograd Areas), Kazakhstan (West Kazakhstan Area) [Spassky, 1940; Platnick \& Ovtsharenko, 1991; Ponomarev \& Tsvetkov, 2004; Piterkina \& Ovtcharenko, 2009; present data]. Crimea is the westernmost point of $T$. strandi distribution.

HABITATS. Sparse forests with Pistacia mutica, steppes and semideserts.

PHENOLOGY. In Crimea: $\sigma^{\top} \sigma^{\top}-$ VI-VIII; + + VII [present data]. A similar phenology for this species was reported from Rostov Area: $\sigma^{7}+$ - VI-VII [Ponomarev \& Tsvetkov, 2004], but in West Kazakhstan (Dzhanibek): $\bigcirc^{7} \bigcirc^{7}$ — IX, males were collected one month later than in Crimea [Platnick \& Ovtsharenko, 1991].

ACKNOWLEDGMENTS. We sincerely thank A.A. Nadolny (Simferopol) and M.K. Yusufova (Mezhdurech'e Vill., Sudak Distr. of Crimea) for providing us with some spiders collected from Crimea; T.V. Piterkina (Moscow), N.Yu. Polchaninova (Kharkiv) and A.V. Ponomarev (Rostov-on-Don) for discussion. We thank P.E. Gol'din (Simferopol) for improving English of the earlier draft and Yu.M. Marusik (Magadan) for some corrections. English of the final drawft was kindly checked by Don Buckle (Saskatoon).

This work of M.K. was supported by the Karadag Nature Reserve. 


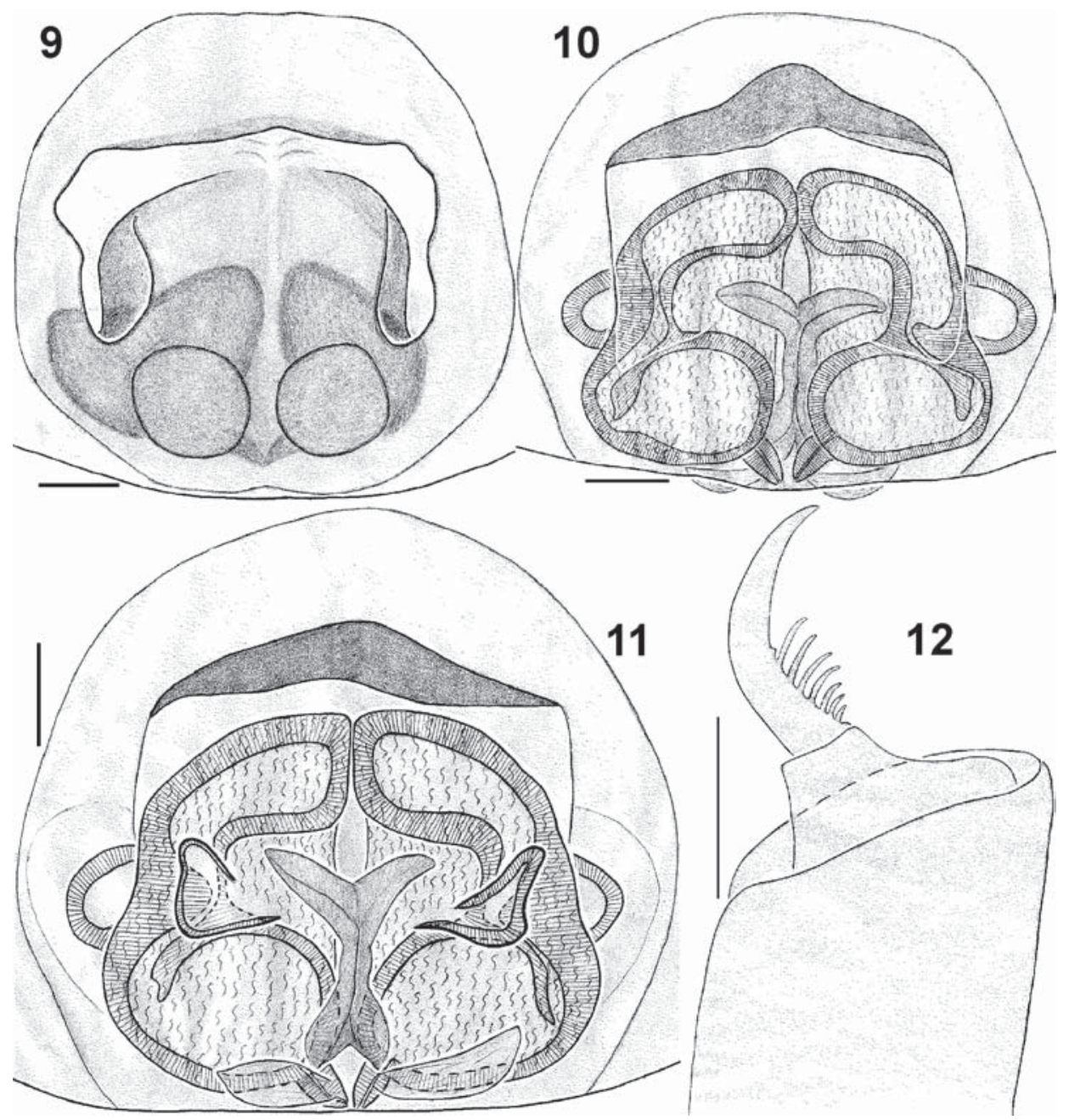

Figs 9-12. Female of Talanites strandi: 9 - epigyne, ventral view; 10 - epigyne, ventral view (after maceration); 11 - epigyne, dorsal view (after maceration); 12 - palpal claw, prolateral view. Scale bars: $0.1 \mathrm{~mm}$.

Рис. 9-12. Самка Talanites strandi: 9 - эпигина, вентрально; 10 - эпигина, вентрально (после мацерации); 11 - эпигина, дорсально (после мацерации); 12 - коготок пальпы, пролатерально. Масштаб: 0,1 мм.

\section{References}

Kovblyuk N.M. 2004. [Catalogue of the spiders (Arachnida: Aranei) of the Crimea, South Ukraine] // Voprosy razvitiya Kryma. Vyp.15. Problemy inventarizatsii krymskoi bioty. Simferopol: Tavriya-Plus. P.211-262 [in Russian], 321 [English summary].

Kovblyuk M.M. 2006. [Gnaphosid spiders (Arachnida: Aranei) in Crimean fauna]. Abstract of $\mathrm{PhD}$ thesis. Kyiv: Institute of Zoology Ukrainian Acad. of Sci. 20 p. [in Ukrainian with Russian and English summary].

Kovblyuk M.M., Nadolny A.A. 2010. Cryptodrassus hungaricus and Leptodrassex memorialis from Crimea (Aranei: Gnaphosidae) // Arthropoda Selecta. Vol.19. No.3. P.189-197.

Kovblyuk M.M., Seyyar O., Demir H., Topcu A. 2009. New taxonomic and faunistic data on the gnaphosid spiders of Turkey (Aranei: Gnaphosidae) // Arthropoda Selecta. Vol.18. No.3-4. P.169-187.

Kovblyuk M.M., Tuneva T.K. 2009. Three interesting species of Gnaphosidae from Crimea (Arachnida: Aranei) // Arthropoda Selecta. Vol.17 (for 2008). No.3-4. P.157-164

Levy G. 1999. Spiders of the genera Anagraphis and Talanites
(Araneae, Gnaphosidae) from Israel // Israel Journal of Zoology. Vol.45. P.215-225

Piterkina T.V., Ovtcharenko V.I. 2009. Fauna and ecology of gnaphosid spiders (Aranei: Gnaphosidae) in clay semidesert of Western Kazakhstan // Arthropoda Selecta. Vol.17. No.3-4. P.175-184.

Platnick N.I. 2012. The world spider catalog, version 12.5. American Museum of Natural History, online at http://research.amnh.org/ iz/spiders/catalog.

Platnick N.I., Ovtsharenko V.I. 1991. On Eurasian and American Talanites (Araneae, Gnaphosidae) // Journal of Arachnology. Vol.19. No.2. P.115-121.

Ponomarev A.V., Tsvetkov A.S. 2004. [The generalized data on spiders (Aranei) of the Nature Reserve «Rostovski»] // Trudy gosudarstvennogo prirodnogo zapovednika «Rostovsky». Vol.3. P.84-104 [in Russian].

Spassky S. 1940. Araneae palaearcticae novae. V // Folia zoologica et hydrobiologica. Vol.10. No.2. P.353-364.

Wunderlich J. 2011. Taxonomy of extant and fossil (Eocene) European ground spiders of the family Gnaphosidae (Araneae), with a key to the genera, and descriptions of new taxa // Beiträge zur Araneologie. Vol.6. P.19-97.

Responsible editor Yu.M. Marusik 